\title{
ARTICLE \\ Bufalin suppresses the migration and invasion of prostate cancer cells through HOTAIR, the sponge of miR-520b
}

\author{
Jian-jun Zhang ${ }^{1,2}$, Xiao-hui Zhou ${ }^{3}$, Yan Zhou ${ }^{2}$, Yong-gang Wang ${ }^{2}$, Bin-zhi Qian ${ }^{4}$, Ai-na He ${ }^{2}$, Zan Shen ${ }^{2}$, Hai-yan Hu ${ }^{2}$ and Yang Yao ${ }^{2}$
}

\begin{abstract}
Bufalin, the major active component of the traditional Chinese medicine ChanSu obtained from the skin and parotid venom glands of toads, has long been known as an anticancer agent. Recent studies show that microRNAs (miRs) are involved in the anticancer activities of bufalin, while long non-coding RNAs (IncRNAs) are known to interact with miRNAs to regulate various biological functions. In this paper, we investigated the possible network related to the antimetastatic effect of bufalin in prostate cancer (PCa) cells. We demonstrated that bufalin $(0.05-10 \mu \mathrm{M})$ dose-dependently suppressed the proliferation of prostate cancer DU145 and PC3 cells with IC $\mathrm{C}_{50}$ values of 0.89 and $1.28 \mu \mathrm{M}$, respectively. Furthermore, bufalin treatment significantly suppressed the cell migration and invasion. To explore the role of IncRNAs in the antimetastatic activity of bufalin, we used an IncRNA microarray and found that HOX transcript antisense RNA (HOTAIR) was the most markedly downregulated IncRNA in bufalin-treated PCa cells. Overexpression of HOTAIR counteracted the suppressing effects of bufalin on DU145 and PC3 cells. We then predicted and verified that HOTAIR upregulated FGFR1 expression by sponging miR-520b in PCa cells. In 40 patients with PCa bone metastasis, we used in situ hybridization or immunohistochemical assay to assess the HOTAIR and FGFR1 expression, which revealed that both HOTAIR and FGFR1 expression were significantly higher in bone metastasis tissues than in the primary PCa tissues. In addition, the level of serum HOTAIR was positively associated with the levels of serum bone metabolic markers (CTx, OST, B-ALP and PINP) and may serve as a reasonable biomarker for PCa bone metastasis. Taken together, this is the first study revealing that HOTAIR promotes PCa bone metastasis, and bufalin may be a promising candidate for the treatment of this disease.
\end{abstract}

Keywords: bufalin; traditional Chinese medicine; prostate cancer; bone metastasis; IncRNA; HOTAIR; miR-520b; FGFR1

Acta Pharmacologica Sinica (2019) 40:1228-1236; https://doi.org/10.1038/s41401-019-0234-8

\section{INTRODUCTION}

Prostate cancer ( $\mathrm{PCa}$ ) is one of the most frequently diagnosed cancers in males, especially in the western world [1, 2]. In PCa patients, the bones are the most frequent, distant metastatic sites, and metastasis to the bones is the first cause of mortality associated with PCa [3]. For metastatic PCa, androgen deprivation therapy (ADT) is the preferred systemic treatment. Unfortunately, most ADT-sensitive patients experience disease progression in a few years, and their prostate cancer eventually evolves into castration-resistant prostate cancer (CRPC) [4]. The prognosis for CRPC remains very poor. Therefore, new strategies that could improve the clinical outcomes of CRPC and PCa bone metastasis urgently need to be explored.

Bufalin is the major active component of the Chinese medicine ChanSu, which is obtained from the skin and parotid venom glands of toads. In recent decades, many studies have corroborated the anticancer properties of bufalin in various cancers, including leukemia, lung cancer, gastric cancer and liver cancer [5-8]. Some studies have also explored the inhibition of proliferation and the apoptosisinducing potential in PCa [9-13]. However, the effects of bufalin on metastatic PCa remain obscure [14]. Recently, several studies have discovered that microRNAs (miRNAs) are involved in the anticancer activities of bufalin $[9,15,16]$. Long-noncoding RNAs (IncRNAs) are known to interact with miRNAs to regulate various biological functions [17-19], and the role of IncRNAs in the anticancer effects of bufalin has not been discussed. In this study, we aimed to identify the possible network that is activated by bufalin in PCa cells, and we conducted a IncRNA microarray analysis in DU145 cells treated with and without bufalin. We found that bufalin significantly inhibited the cell proliferation, migration and invasion abilities of DU145 and PC3 cells. HOX transcript antisense RNA (HOTAIR) was found to be a key IncRNA in the effects of bufalin on PCa cells. We proved that HOTAIR was upregulated in PCa bone metastatic tissues compared with the expression in primary PCa tissues, and the serum level of HOTAIR was positively associated with serum levels of bone metabolic markers, which are significantly elevated in patients with PCa bone metastasis [20]. For the first time, we propose that the overexpression of HOTAIR promotes $\mathrm{PCa}$ bone metastasis.

\section{MATERIALS AND METHODS}

Cells culture and proliferation assay

The CRPC cell lines DU145 and PC3 were obtained from the Cell Bank of Type Culture Collection of the Chinese Academy of Sciences (Shanghai, China). The cells were cultured in DMEM (Invitrogen,

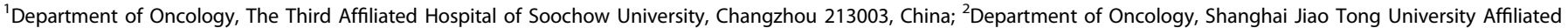

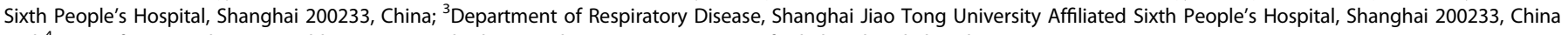
and ${ }^{4}$ Centre for Reproductive Health, Queens Medical Research Institute, University of Edinburgh, Edinburgh EH16 4TJ, UK

Correspondence: Hai-yan Hu (xuri1104@163.com) or Yang Yao (yangyao_6@hotmail.com)

These authors contributed equally: Jian-jun Zhang, Xiao-hui Zhou

Received: 17 November 2018 Accepted: 30 March 2019

Published online: 26 April 2019 
Carlsbad, CA, USA) supplemented with $10 \%$ fetal bovine serum (FBS, HyClone, Logan, UT, USA), $100 \mu \mathrm{g} / \mathrm{mL}$ streptomycin, and $100 \mathrm{U} / \mathrm{mL}$ penicillin at $37{ }^{\circ} \mathrm{C}$ in an incubator with a constant air flow of $5 \% \mathrm{CO}_{2}$ and $95 \% \mathrm{O}_{2}$ and were routinely passaged every 2-3 days. Bufalin (Sigma-Aldrich, St Louis, MO, USA) was dissolved to a concentration of $50 \mathrm{mM}$ in dimethyl sulfoxide (DMSO, Sigma-Aldrich, St Louis, MO, USA) and was stored at $-80^{\circ} \mathrm{C}$. The final concentrations were $0.05-10 \mu \mathrm{M}$. The cells that were treated with the vehicle only (DMSO, $0.1 \%$ in medium) served as the blank control. A Cell Counting Kit- 8 (CCK-8, Dojindo Molecular Technologies, Dojindo, Japan) was used for the cell proliferation assay and was performed according to the protocol. The absorbance value was measured for each well at a wavelength of $450 \mathrm{~nm}$, and the reference wavelength was set at 630 $\mathrm{nm}$. The experiments were repeated three times, and the percentage of bufalin-induced proliferation inhibition was calculated by comparison to that in the untreated control cells.

Wound healing assay

A wound healing assay was used to detect the migration ability of PCa cells. DU145 and PC3 cells in a 6-well plate were carefully scratched using $200 \mu \mathrm{L}$ sterile pipette tips. Then, the wells were washed twice and were incubated with fresh complete medium (DMEM) with or without half of the $\mathrm{IC}_{50}$ of bufalin. Images were taken at $24 \mathrm{~h}$ to evaluate the width of the wound using a microscope ( $\times 4$ objective) $(1 \times 71$, Olympus).

Transwell assay

Boyden chamber (Corning Inc., Corning, NY, USA) assays were used for Transwell assays. Generally, $1 \times 10^{5}$ cells are seeded into the Matrigel-coated upper chamber. The concentrations of bufalin in both the upper and lower chambers were equal to those used in the wound healing assay. After culturing for $8 \mathrm{~h}$, the cells that migrated through the filter were fixed with $4 \%$ paraformaldehyde and were stained with a $0.1 \%$ crystal violet solution. The invading cells were counted in five random fields under the $\times 20$ objective, and images were obtained.

Microarray analysis of the IncRNA profiles

We analyzed the change in the IncRNA profiles between DU145 cells that were treated with and without the $\mathrm{IC}_{50}$ of bufalin for $24 \mathrm{~h}$ using Agilent human IncRNA $(4 \times 180 \mathrm{~K})$ (Bohao Biocompany, Shanghai, China). For this array, the global expression of IncRNAs was detected together after normalization with the log2 scale. We identified the differentially expressed IncRNAs with a discriminating parameter of $q<0.05$ [21].

Bioinformatics analysis of the HOTAIR, miR-520b and FGFR1 binding sites

We scanned the HOTAIR sequence to search for matched miRNAs with complementary binding sites using our designed software [21]. Furthermore, we predicted the direct miRNA targets using the TargetScan website.

Luciferase reporter assay

We constructed the wild-type (WT) or mutant (MUT) HOTAIR/ FGFR1 psiCheck2 dual-luciferase plasmid that contained the binding site of miR-520b. Then, the plasmid was transfected into
HEK293T cells using Lipofectamine 3000. Renilla luciferase assays were performed according to the protocol (Promega, Madison, WI, USA).

\section{qRT-PCR assay}

The HOTAIR, miR-520b and FGFR1 levels were detected using a PrimeScript RNA RT-PCR Kit (Sangon Biotech, Shanghai, China) following the manufacturer's protocol. The primer pairs are shown in Table 1. The relative expression levels of HOTAIR, miR-520b and FGFR1 were calculated using the $2^{-\Delta \Delta C \mathrm{t}}$ method.

Western blot assay

The proteins in the cells were quantified using a BCA Protein Assay Reagent Kit (Thermo Fisher, USA). The rabbit monoclonal FGRF1 antibody was purchased from Boster Biology Technology (Wuhan, China), and the band density was quantified using ImageJ lab software.

RNA immunoprecipitation (RIP) assay

An RIP assay was performed to further verify the sponge function of HOTAIR using an EZ-Magna RIP kit (Millipore, USA) as previously described [21]. Briefly, after the overexpression of HOTAIR for $24 \mathrm{~h}$, the total protein was extracted from DU145 and PC3 cells and was incubated with an IgG or AGO2 antibody. Subsequently, the relative levels of HOTAIR and miR-520b were analyzed by qRT-PCR.

Construction of the plasmids and transfection

The HOTAIR overexpression pcDNA3.1 plasmid and the siRNAHOTAIR pLK0.1, miR-520b-MIMIC and miR-520b-AMO (anti-microRNA oligonucleotide) plasmids were constructed by Jierui Bio Com (Shanghai, China). The plasmids were transfected into DU145 or PC3 cells using Lipofectamine 3000 (Invitrogen, Carlsbad, CA, USA) according to the manufacturer's protocol.

In situ hybridization (ISH) assay for HOTAIR

Paired paraffin sections of primary prostate tumors and bone metastases from 40 PCa patients were collected. This study was approved by the Shanghai Jiao Tong University Affiliated Sixth People's Hospital ethics committee. The ISH assay for HOTAIR was performed as previously described [22]. The sections were stained and scored on a three-tiered scoring system using the following criteria: $0=$ negative; $1=$ equivocal/uninterpretable; $2=$ weak positive; $3=$ strong positive. The expression was scored by two independent observers, and the mean value of both scores was used.

Immunohistochemical (IHC) assay for FGFR1

The IHC assay was performed as previously described [23]. Briefly, after deparaffinization and antigen retrieval, the nonspecific binding was blocked by incubating the specimens for $30 \mathrm{~min}$ in $10 \%$ bovine serum albumin. Subsequently, a rabbit anti-FGFR1 antibody (Boster Biology Technology, Wuhan, China) was used for IHC staining. The specimens were then incubated with the secondary antibody and Envision Plus Dual Link-labeled polymer (DAKO, USA). Then, they were incubated with the chromogen diaminobenzidine. FGFR1 staining was scored using the following previously described criteria: $0=$ negative; $1=$ equivocal/uninterpretable; $2=$ weak positive; $3=$ strong positive [22].

\begin{tabular}{|lll|}
\hline Table 1. & The qRT-PCR primers & \\
\hline & Forward & Reverse \\
\hline HOTAIR & $5^{\prime}$-GGTAGAAAAAGCAACCACGAAGC-3' & $5^{\prime}$-ACATAAACCTCTGTCTGTGAGTGCC-3' \\
FGFR1 & $5^{\prime}$-GGCTACAAGGTCCGTTATGCC-3' & $5^{\prime}$-GATGCTGCCGTACTCATTCTC -3' \\
U6 & $5^{\prime}$-CTCGCTTCGGCAGCACA-3' & $5^{\prime}$-AACGCTTCACGAATTTGCGT-3' \\
GAPDH & $5^{\prime}$-ACAACTTTGGTATCGTGGAAGG-3' & $5^{\prime}$-GCCATCACGCCACAGTTTC-3' \\
\hline
\end{tabular}




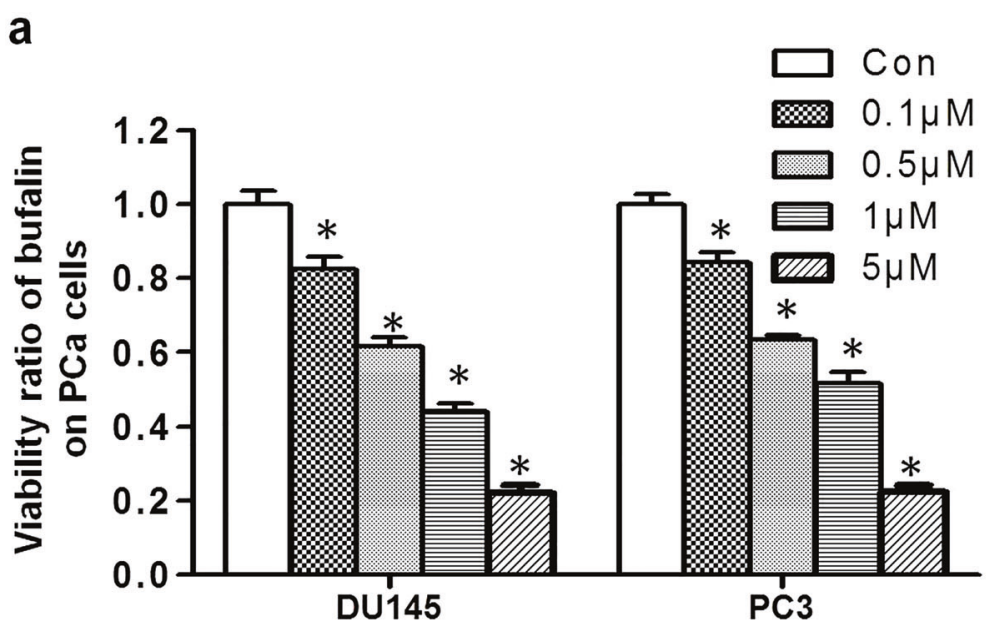

C
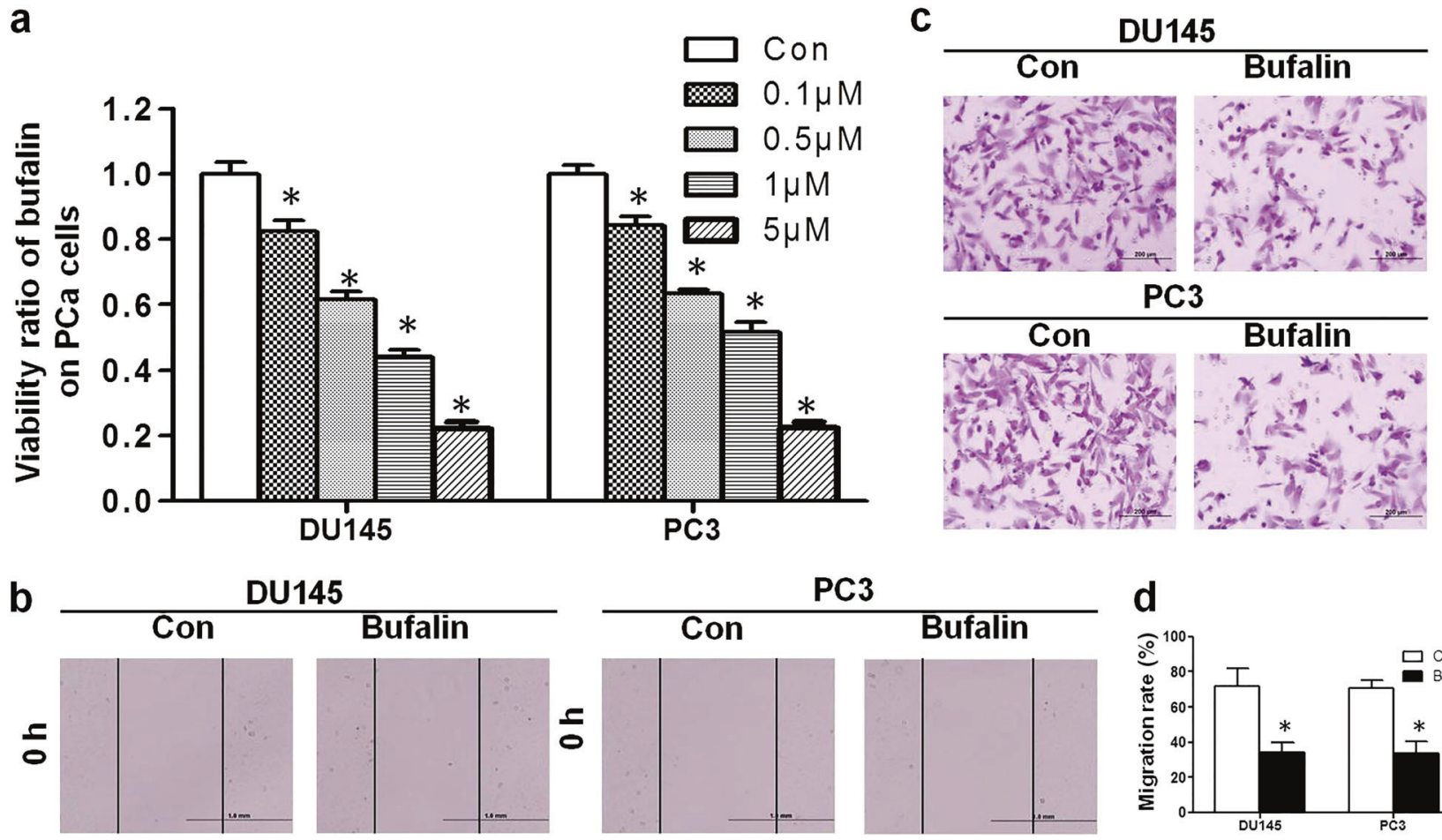

DU145
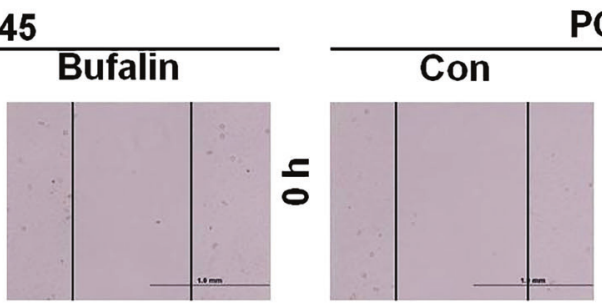

PC3
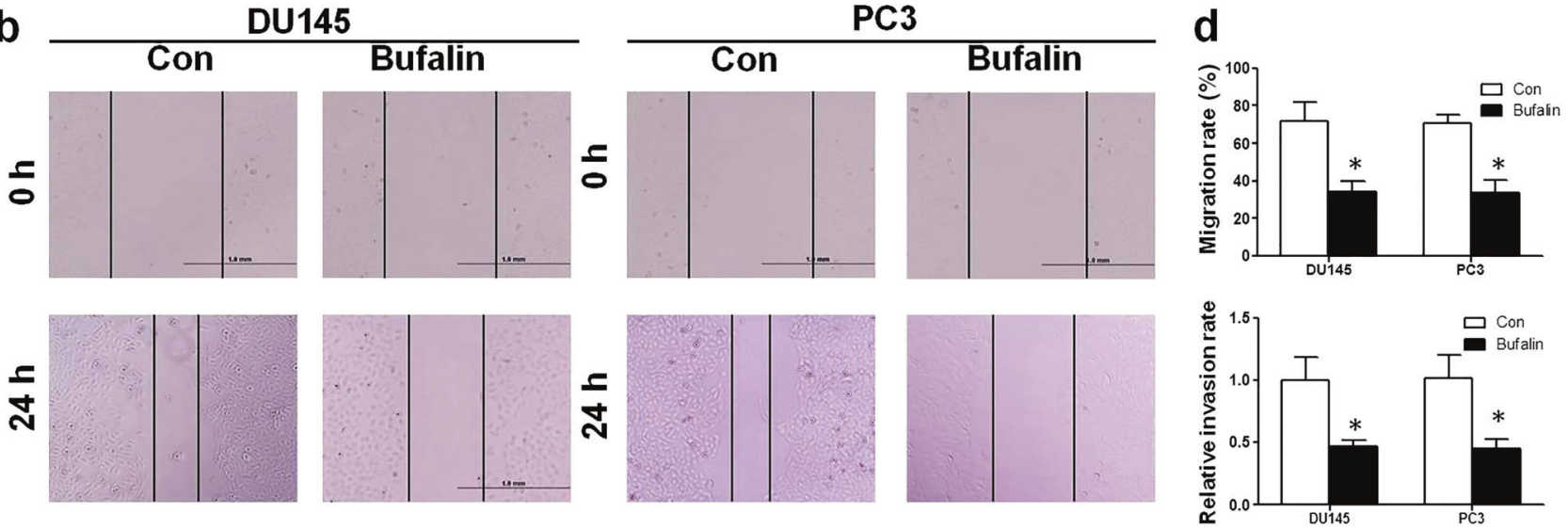

Fig. 1 Bufalin regulates the cell proliferation, migration and invasion of PCa cells. a After exposure to various concentrations $(0.05-10 \mu M)$ of bufalin for $24 \mathrm{~h}$, the viability of DU145 and PC3 cells was assessed with a CCK-8 assay. The proliferation ratio decreased dose-dependently. b After incubation with half of the $\mathrm{IC}_{50}$ of bufalin, the wound healing assay indicated that bufalin impaired wound healing; the results were almost half the value of the control group. c Transwell assays showed that the invasion rates of PCa cells decreased sharply in the bufalintreated group. $\mathbf{d}$ Comparisons of the migration rates and relative invasion rates between the bufalin and control groups are shown $(* P<0.05$ compared with the control)

Measurement of serum bone metabolic markers

Serum samples from 80 PCa patients with and without bone metastasis ( $n=40$ cases for each group) were collected and stored at $-80^{\circ} \mathrm{C}$. The serum levels of C-telopeptide-cross-linked type I collagen (CTx), osteocalcin (OST), bone-specific alkaline phosphatase (B-ALP) and procollagen type I N-terminal propeptide (PINP) were measured as previously reported [20].

\section{Statistical analysis}

The data analysis was performed using Statistical Package for the Social Sciences version 19.0. $P$ values were two-tailed, and $P$ $<0.05$ was considered statistically significant. Continuous variables are presented as the mean $\pm S D$. The qualitative variables are expressed as absolute and relative frequencies. The Wilcoxon rank-sum test was used to compare the ISH and IHC scores from the paired paraffin sections. A $t$-test was used to evaluate the differences in the serum markers between the two groups. Spearman correlation analysis was used to analyze the association between the serum levels of HOTAIR and the bone metabolic markers. A receiver-operating characteristics (ROC) curve was plotted to determine how well the serum levels of HOTAIR and the bone metabolic markers discriminated between the PCa patients with and without bone metastasis. An area-under-the-curve (AUC)-ROC value of $>0.7$ was taken to indicate reasonable biomarker performance. The sensitivity and specificity of each marker were calculated based on the cutoff value.

\section{RESULTS}

Bufalin suppressed the viability and invasion of PCa cells in vitro The proliferation assay revealed that bufalin significantly inhibited the proliferation of DU145 and PC3 cells in a dose-dependent manner (Fig. 1a). The $\mathrm{IC}_{50}$ at $24 \mathrm{~h}$ was determined to be 0.89 and $1.28 \mu \mathrm{M}$ for the DU145 and PC3 cells, respectively. Wound healing assays and Transwell assays were performed to determine the antimetastatic activity of bufalin. As shown in Fig. 1b, d, compared with the migration rates of the control group, the migration rates of the DU145 and PC3 cells that were treated with bufalin were markedly decreased from $72.1 \%$ to $34.3 \%$ and from $70.8 \%$ to $33.6 \%$, respectively. In the Transwell assay, bufalin inhibited PCa cell invasion through the Transwell membrane compared with that in the control group (Fig. 1c, d).

Bufalin downregulated HOTAIR expression

To explore the role of IncRNAs in the activity of bufalin, we screened the functional IncRNA change in DU145 cells that were exposed to the $\mathrm{IC}_{50}$ of bufalin using a IncRNA microarray. Among the changed IncRNAs (Fig. 2a), we selected HOTAIR for further study because it was the most markedly downregulated IncRNA, 
a

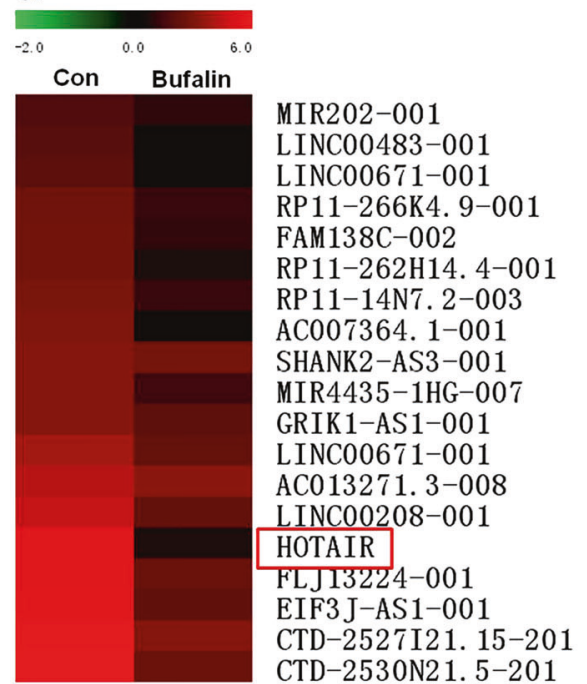

b

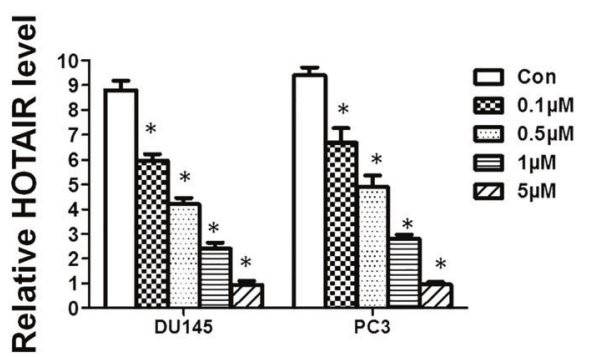

Fig. 2 Change in IncRNA profiles in PCa cells treated with bufalin. a The changes in the IncRNA profiles in DU145 cells after bufalin treatment indicated that the most markedly downregulated IncRNA was HOTAIR. b As detected by qRT-PCR, the HOTAIR levels in the DU145 and PC3 cells significantly decreased by $2-6$-fold after treatment with bufalin $\left({ }^{*} P<0.05\right.$ compared with the control)

and the result can be repeated and verified by qRT-PCR. Furthermore, we verified that the level of HOTAIR in DU145 and PC3 cells was reduced in a dose-dependent manner (Fig. 2b).

HOTAIR complementary sequence binds to miR-520b as a competing endogenous RNA (ceRNA)

To explore the downstream signaling pathway of HOTAIR in the tumor suppressor function of bufalin, we identified the potential sponge function of HOTAIR on miRNAs. We found that there was one seed sequence of miR-520b that was complementary to the $3^{\prime}$ UTR of HOTAIR (Fig. 3a). A dual-luciferase reporter assay was used to confirm the direct correlation between miR-520b and HOTAIR. The transfection of both the WT HOTAIR and miR-520b-MIMIC plasmids into DU145 cells decreased the reporter activity (Fig. 3b). AGO2 is a key component of RNA-induced silencing complexes, which are formed when miRNAs combine with IncRNAs [24]. The results of the RIP assay showed that HOTAIR and miR-520b were significantly higher in the AGO2-containing miRNA complexes than they were in the control IgG immunoprecipitation complexes, further verifying the sponge function of HOTAIR for miR520b (Fig. 3c).

FGFR1 was a direct target gene of miR-520b

The potential targets of miR-520b were predicted by a TargetScan analysis. There was one seed sequence for miR-520b in the $3^{\prime}$ UTR of FGFR1, a well-known oncogenic gene in PCa [25] (Fig. 4a). Similarly, to confirm the direct interaction between miR-520b and FGFR1, luciferase reporter plasmids were constructed for both WT FGFR1 and MUT FGFR1. As displayed in Fig. 4b, the luciferase activity of WT FGFR1 but not MUT FGFR1 inversely correlated with the miR-520b expression levels. To validate the regulatory effect of miR-520b on FGFR1, we transfected miR-520b-MIMIC and miR-520b-AMO into DU145 cells. Both the mRNA and protein levels of FGFR1 were inversely correlated with the miR-520b levels (Fig. 4c, d).

HOTAIR inhibits miR-520b expression and upregulates FGFR1 expression

As mentioned above, HOTAIR interacts with miR-520b directly in PCa cells. Next, we investigated whether HOTAIR affects the expression of miR-520b and its target gene FGFR1. After the overexpression or silencing of HOTAIR in PCa cells (Fig. 5a), the expression levels of miR-520b and FGFR1 were evaluated using qRT-PCR. The results revealed that the miR-520b expression level negatively correlated with the HOTAIR expression level in DU145 and PC3 cells (Fig. 5b). Conversely, the mRNA and protein levels of FGFR1 positively correlated with the HOTAIR expression level (Fig. 5c, d).

Overexpression of HOTAIR reversed the bufalin effect

To further determine whether HOTAIR is a key mediator of the bufalin effect, we overexpressed HOTAIR in PCa cells that were treated with bufalin. As expected, the viability of DU145 and PC3 cells significantly increased in the HOTAIR overexpression group compared with that in the control group (Fig. 6a). In addition, the wound healing assay and the Transwell assay revealed that the overexpression of HOTAIR reversed the suppressive effect of bufalin on DU145 and PC3 cells (Fig. 6b-d). Moreover, the increase of miR-520b in both PCa cell types after exposure to bufalin was counteracted by the overexpression of HOTAIR (Fig. 6e). For FGFR1, the mRNA and protein levels were much higher in the HOTAIR overexpression group than the levels in the control group (Fig. 6f, g).

HOTAIR and FGFR1 were upregulated in PCa bone metastatic tissues, and serum HOTAIR was positively associated with bone metabolic markers

HOTAIR was reported to promote cancer metastasis in a variety of cancers. In this study, we investigated the potential function of HOTAIR in PCa bone metastasis for the first time. As shown in Fig. $7 \mathrm{a}$, we found that bone metastatic tissues had significantly increased HOTAIR expression compared with the primary PCa tissues; the mean ISH scores of HOTAIR were 2.08 and 1.38, respectively $(P<0.05)$. Accordingly, as a target gene in the HOTAIR/miR-520b/FGFR1 axis, FGFR1 expression in bone metastatic tissues was significantly higher than that in the primary $\mathrm{PCa}$ tissues, and the mean IHC scores of FGFR1 were 1.97 and 1.15, respectively $(P<0.05$, Fig. $7 b)$. Serum bone metabolic markers are found to be elevated in PCa patients with bone metastasis and are often used to diagnose bone metastasis and to monitor disease progression [20, 26-28]. Likewise, we found that the serum levels of bone metabolic markers, including CTx, OST, B-ALP and PINP, in PCa patients with bone metastasis were significantly higher than those in patients without bone metastasis (Table 2). Furthermore, the serum HOTAIR levels were positively associated 
WT HOTAIR

miR-520b

MUT HOTAIR

\section{5'...AUUGGGUAGGUAUGCACUUUGU... 3' 3'...UGAGAUUUCCCUUCGUGAAACA... 5' \\ 5'...AUUGGGUAGGUAUGCACAAAGU... 3'}
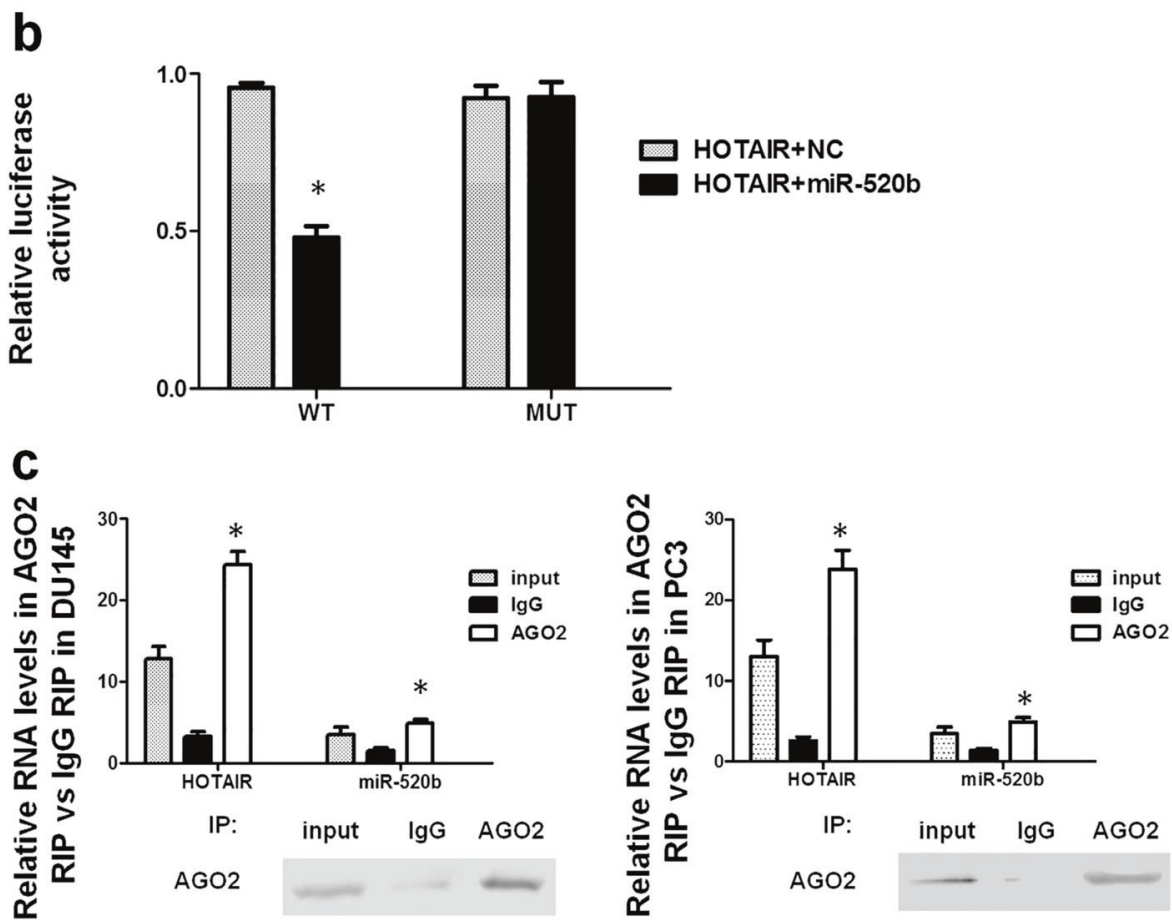

Fig. 3 HOTAIR regulates miR-520b as a competing endogenous RNA. a The binding region between HOTAIR and miR-520b is highlighted in red. The MUT sequence of HOTAIR is highlighted in yellow. b The luciferase activity decreased significantly in the WT HOTAIR transfection group compared with that in the control, and the luciferase activity for the MUT HOTAIR group did not change significantly compared with that in the control $(* P<0.05$ compared with the control). c After the overexpression of HOTAIR in DU145 and PC3 cells, the total protein was incubated with an IgG or AGO2 antibody, and the HOTAIR and miR-520b expression levels were significantly higher in the AGO2 group than they were in the IgG group ( ${ }^{*} P<0.05$ compared with the IgG group)

with the levels of the aforementioned bone metabolic markers in PCa patients $(P<0.05$, Fig. 7c). All of the AUC-ROC values for the serum HOTAIR and bone metabolic markers were $>0.7$ (Fig. $7 d$ ), which indicates a reasonable biomarker performance. Notably, the serum HOTAIR marker had comparable sensitivity and specificity to those of the bone metabolic markers. The respective sensitivity and specificity of the serum markers were as follows: HOTAIR, 70\% and $72.5 \%$ at a cutoff value of $0.39 \mathrm{ng} / \mathrm{mL}$; CTx, 77.5 and $75 \%$ at a cutoff value of $599.9 \mathrm{ng} / \mathrm{mL}$; OST, $70 \%$ and $75 \%$ at a cutoff value of $23.89 \mathrm{ng} / \mathrm{mL}$; B-ALP, $67.5 \%$ and $90 \%$ at a cutoff value of $19.39 \mathrm{ng} /$ $\mathrm{mL}$; and PINP, $77.5 \%$ and $80 \%$ at a cutoff value of $69.2 \mathrm{ng} / \mathrm{mL}$. These results suggest that the serum HOTAIR marker might serve as a reasonable biomarker for PCa bone metastasis.

\section{DISCUSSION}

Bufalin exhibits significant anti-tumor activities in a variety of cancers. However, the antimetastatic activity of bufalin in prostate cancer has yet to be determined. The migratory and invasive behavior of cancer cells is the foundation for tumor metastasis. In this study, we investigated whether bufalin can suppress the migratory and invasive abilities of DU145 and PC3 cells using a wound healing assay and a Transwell assay. The results suggest that bufalin significantly inhibited the migration and invasion of PCa cells.

The dysregulation of IncRNAs has been implicated in various aspects of cellular homeostasis, such as proliferation, apoptosis, mobility, invasion, and metastasis [29]. To identify the possible role of IncRNAs regulated by bufalin in PCa cells, we performed a IncRNA microarray in DU145 cells treated with and without bufalin. Our findings suggest that HOTAIR is a key IncRNA that is involved in the effects of bufalin on PCa cells, and this result was confirmed by qRT-PCR in DU145 and PC3 cells. HOTAIR is a recently discovered oncogenic IncRNA that is associated with a poor prognosis for various malignancies, including prostate cancer [22, 30-32]. Oliveira described that HOTAIR genetic variants were significantly associated with a shorter biochemical recurrence-free survival in patients with pT3-stage PCa [33]. Chang et al. found that HOTAIR promotes neuroendocrine differentiation in CRPC [34]. Similarly, Zhang et al. reported that the overexpression of HOTAIR enhances the proliferation and invasion of castrationresistant cells, and the levels of HOTAIR constantly increase in LNCaP cell lines treated with enzalutamide [35]. HOTAIR has been known as a IncRNA that promotes cancer metastasis from the time it was discovered [36]: HOTAIR was found to promote metastasis in a variety of cancers, such as breast cancer, non-small-cell lung cancer, colorectal cancer and hepatocellular carcinoma $[22,31,37,38]$. However, to our knowledge, no study has explored the role of HOTAIR in PCa bone metastasis. Although comparisons between the primary PCa tissues and PCa bone metastatic tissues have been made in previous reports $[39,40]$, comparisons between matched pairs are scarce and are even rarer for comparisons of PCa IncRNAs. In this study, we proved that HOTAIR was upregulated in PCa bone metastatic tissues compared 
a

$$
\begin{array}{rr}
\text { Position 1311-1317 of FGFR1 } & \text { 5'...CACGCCUGUAAUCCCAGCACUUU... } \\
\text { hsa-miR-520b } & \text { 3'...GGGAGAUUUUCCUUCGUGAAA... } \\
\text { MUT FGFR1 } & 5 \text { '...CACGCCUGUAAUCCCAGCACAAU... }
\end{array}
$$
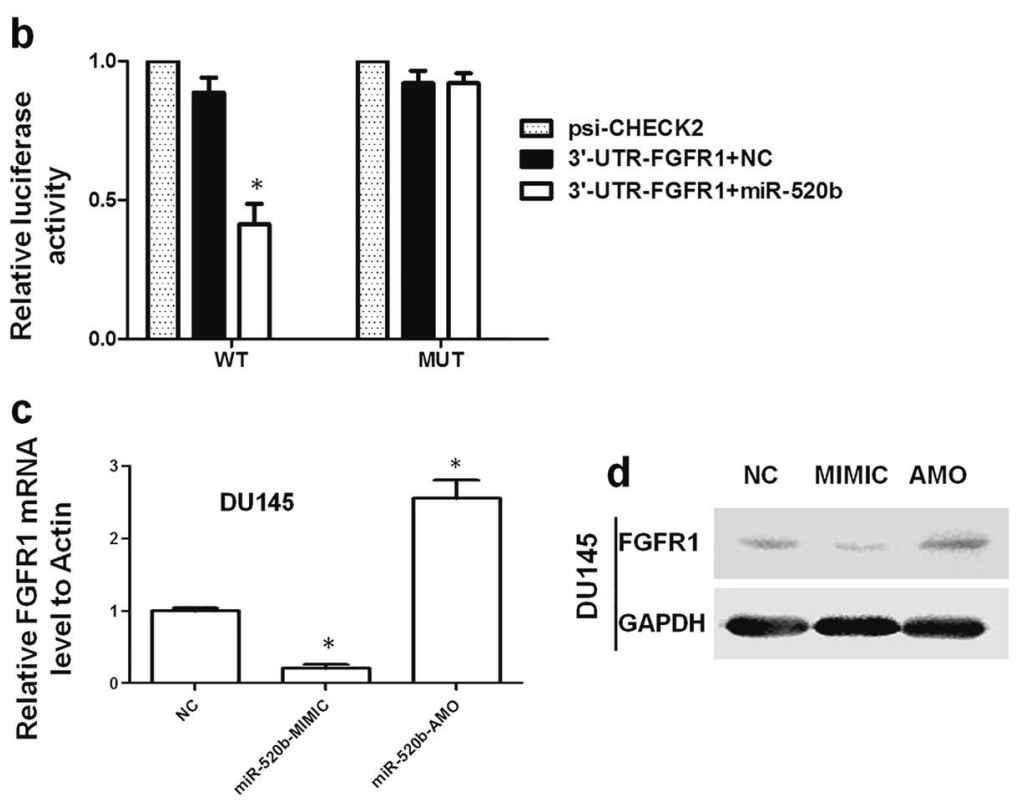

Fig. 4 FGFR1 is a direct target gene of miR-520b. a The binding region between FGFR1 and miR-520b is highlighted in red. The MUT sequence of FGFR1 is highlighted in yellow. $\mathbf{b}$ The luciferase activity decreased significantly in the WT FGFR1 transfection group compared with that in the control, and the luciferase activity for the MUT FGFR1 group did not change significantly compared with that in the control $(* P<0.05$ compared with the control). c After transfection with miR-520b-MIMIC or miR-520b-AMO in DU145 cells, the FGFR1 mRNA expression level negatively correlated with the level of miR-520b $\left({ }^{*} P<0.05\right.$ compared with the control). $\mathbf{d}$ The FGFR 1 protein level was the same as the mRNA level
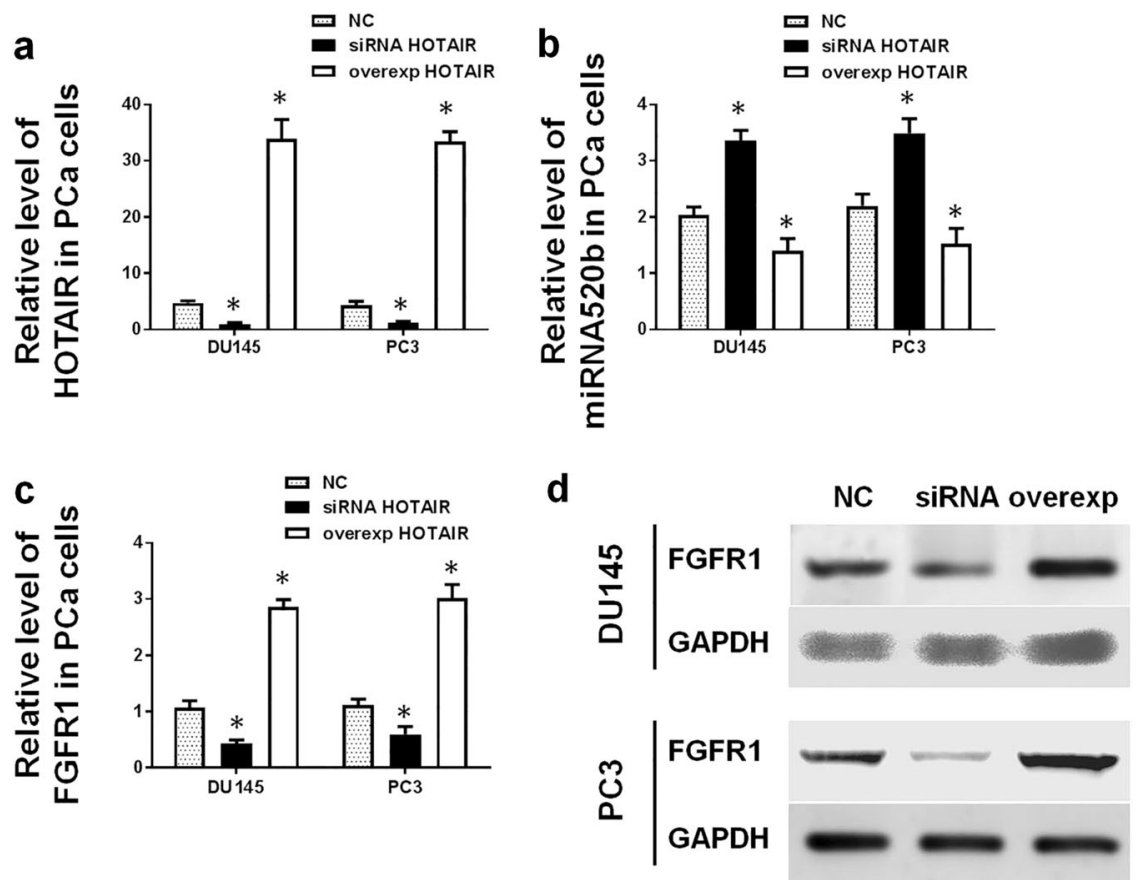

Fig. 5 HOTAIR downregulates miR-520b expression and upregulates FGFR1 expression. a The HOTAIR levels in DU145 and PC3 cells were evaluated by qRT-PCR, and the levels increased significantly in the overexpression group but decreased dramatically in the silencing group $\left({ }^{*} P<0.05\right.$ compared with the control). $\mathbf{b}$ After the overexpression or silencing of HOTAIR in DU145 and PC 3 cells, the miR-520b level negatively correlated with the level of HOTAIR ( ${ }^{*} P<0.05$ compared with the control). (c, d) After the overexpression or silencing of HOTAIR in DU145 and PC3 cells, the mRNA (c) and protein levels (d) of FGFR1 positively correlated with the levels of HOTAIR $\left({ }^{*} P<0.05\right.$ compared with the control) 

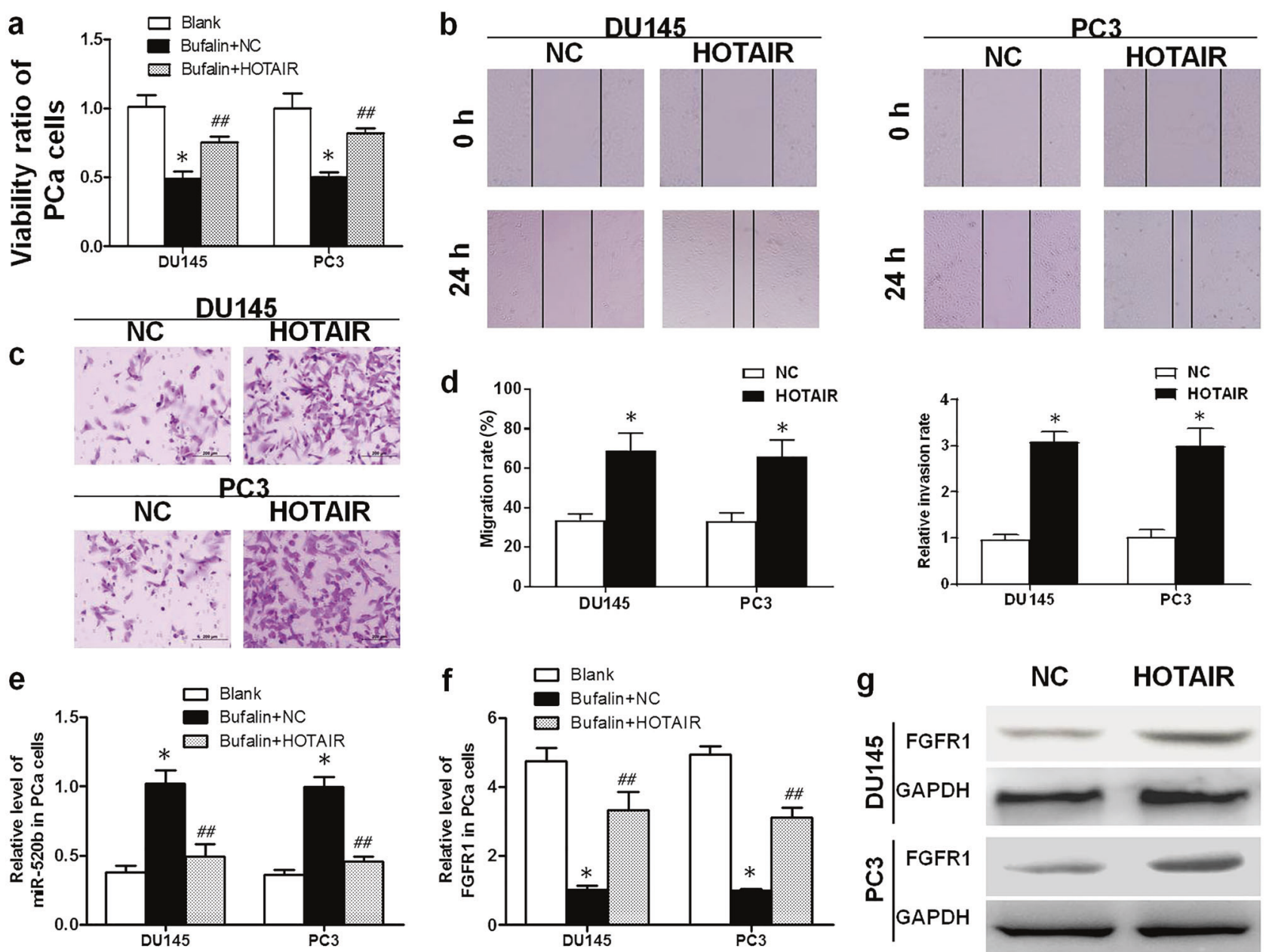

NC HOTAIR

Fig. 6 The HOTAIR/miR-520b/FGFR1 axis plays an important role in the effects of bufalin on PCa cells. a The viability ratio of DU145 and PC3 cells in the HOTAIR overexpression group was significantly higher than that in the NC group after bufalin treatment $\left({ }^{*} P<0.05\right.$ compared with the blank group, ${ }^{\# \#} P<0.05$ compared with the bufalin + NC group). b The wound healing assay revealed that the overexpression of HOTAIR reversed the suppressive effect of bufalin on the migration of PCa cells. c The Transwell assay demonstrated that HOTAIR significantly promoted PCa cell invasion. d Comparisons of the migration rates and relative invasion rates between the NC and HOTAIR groups are shown $\left({ }^{*} P<0.05\right)$. e Bufalin upregulated the relative level of miR-520b in PCa cells, and this effect was reversed by the overexpression of HOTAIR ( ${ }^{*} P<0.05$ compared with the blank group, ${ }^{\# \#} P<0.05$ compared with the bufalin + NC group). $\mathbf{f}, \mathbf{g}$ The overexpression of HOTAIR counteracted the downregulation effects of bufalin on expression of FGFR1 at both the mRNA (f) and protein levels (g) $(* P<0.05$ compared with the blank group, ${ }^{\# \#} P<0.05$ compared with the bufalin $+N C$ group)

with the expression in matched primary PCa tissues by an ISH assay, and the serum level of HOTAIR was positively associated with that of bone metabolic markers. This is the first study to indicate the involvement of HOTAIR in PCa bone metastasis. Our study suggests that HOTAIR is a promising, potential biomarker and therapeutic target for PCa bone metastasis.

With regard to the mechanisms that IncRNAs use to regulate various biological functions, studies have demonstrated that IncRNAs are able to function as a sponge of miRNAs and are able to rescue the miRNA-targeted messenger RNAs [17]. This socalled ceRNA system further complicates the molecular mechanisms of tumorigenesis, cancer development and metastasis [18]. Zheng et al. reported that HOTAIR endogenously competes with miR-148a to regulate DLGAP1 in head and neck tumor cells [19]. Chang et al. found that HOTAIR regulates CCND1 and CCND2 expression by sponging miR-206 in ovarian cancer cells [41]. Hong et al. described that HOTAIR can regulate the HIF-1a/AXL axis in renal cell carcinoma through the inhibition of miR-217 [42]. Similarly, we found that HOTAIR promoted PCa cell migration and invasion by sponging miR-520b, and this ceRNA mechanism was validated by a dual-luciferase reporter assay and an RIP assay. We observed a negative correlation between the levels of HOTAIR and the levels of miR-520b. Further studies are needed to investigate the downstream genes of HOTAIR/miR-520b in PCa.

In this study, we used bioinformatics analyses to predict that FGFR1 is a target gene of miR-520b and validated this result by a dual-luciferase reporter assay. Moreover, HOTAIR can regulate FGFR1 expression by sponging miR-520b in vitro. FGFR1 is a potential target for cancer treatment because of its prominent role in tumorigenesis and cancer progression [25]. FGFR1 expression is significantly higher in poorly differentiated PCa cells compared with the expression in well-differentiated PCa cells [43]. Similarly, we found that FGFR1 expression was higher in bone metastatic tissues than it was in primary PCa tissues.

To verify whether the HOTAIR/miR-520b/FGFR1 axis plays an important role in the effects of bufalin on PCa cells, the reverse test was performed. As expected, after overexpressing HOTAIR in PCa cells, the effects of bufalin on cell proliferation, migration, and invasion, and on the expression of miRNA-520b and FGFR1 were reversed significantly.

In summary, our study is the first demonstration that IncRNAs participate in the anticancer effects of bufalin. The HOTAIR/miR- 


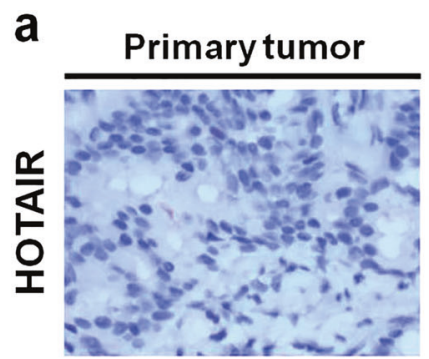

b

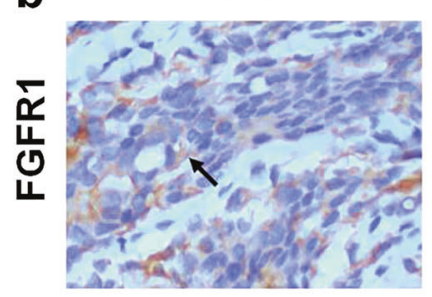

$+$

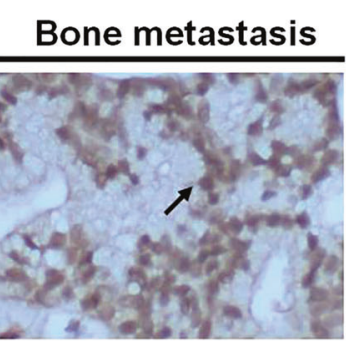

$++$

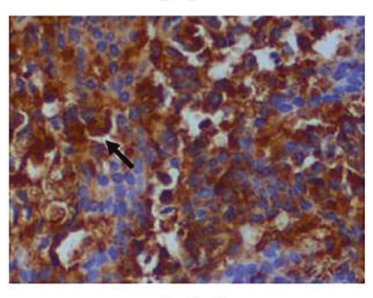

$+++$
C
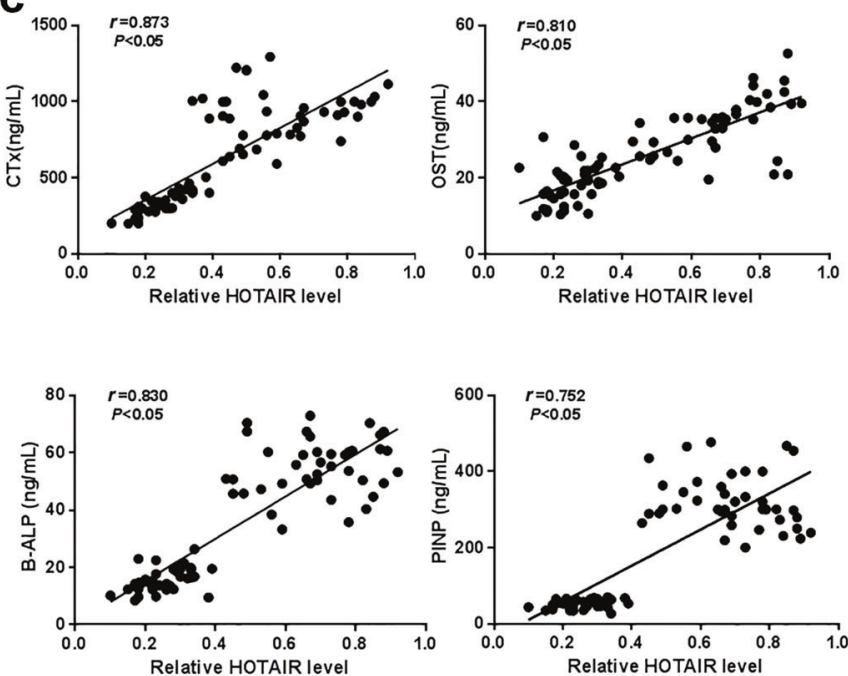

d
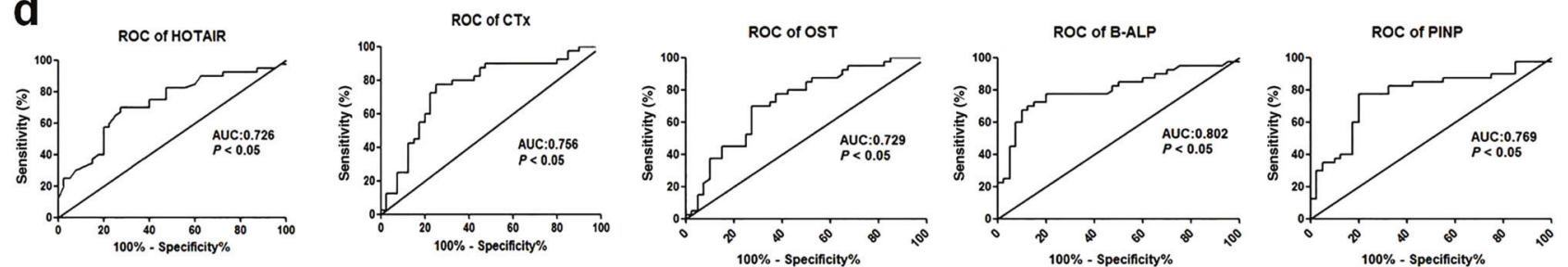

Fig. 7 HOTAIR and FGFR1 were upregulated in PCa bone metastatic tissues. a Representative ISH images of HOTAIR are shown. HOTAIR expression (shown by the black arrow) in bone metastatic tissues was significantly higher than in the expression in the matched primary PCa tissues (ISH, 400x, $n=40)$. b Similar to the HOTAIR expression levels, bone metastatic tissues had a significantly higher FGFR1 expression level (shown by the black arrow) than that in the matched primary PCa tissues (IHC, 400x, $n=40)$. c The serum HOTAIR expression level was positively associated with the levels of bone metabolic markers in PCa patients $(P<0.05, n=80)$. $\mathbf{d}$ The ROC curves of the serum HOTAIR and bone metabolic marker levels are shown

Table 2. The levels of serum HOTAIR and bone metabolic markers in PCa patients with and without bone metastasis

\begin{tabular}{llllrr}
\hline Group & HOTAIR & CTx $(\mathrm{ng} / \mathrm{mL})$ & OST $(\mathrm{ng} / \mathrm{mL})$ & B-ALP $(\mathrm{ng} / \mathrm{mL})$ & PINP $(\mathrm{ng} / \mathrm{mL})$ \\
\hline Metastasis group & $0.69 \pm 0.14^{* * *}$ & $903.03 \pm 174.34^{* * *}$ & $33.96 \pm 7.52^{* * *}$ & $54.79 \pm 9.79^{* * *}$ & $320.49 \pm 71.66^{* * *}$ \\
Non-metastasis group & $0.25 \pm 0.07$ & $338.00 \pm 76.40$ & $17.96 \pm 5.20$ & $15.84 \pm 4.16$ & $52.89 \pm 11.46$ \\
\hline${ }^{* * *} P<0.001$ compared with non-metastasis group & & & \\
\hline
\end{tabular}

520b/FGFR1 axis plays an important role in the effects of bufalin on PCa cells. Moreover, for the first time, we provide clinical evidence that the overexpression of HOTAIR promotes PCa bone metastasis.

\section{ACKNOWLEDGEMENTS}

This work was supported by grants from the National Natural Science Foundation of China (No 81503396), the Shanghai Science and Technology Committee (No. 17411950300), the National Key Research Project of Science and Technology Ministry (No. 2016YFC0106204), the Shanghai Senior Integrative Chinese and Western Medicine Talents Program (ZY(2018-2020)-RCPY-2017, Pujiang Talent project (No. 17PJ1407600), and Talent project of the Sixth People`s Hospital of Shanghai (No. ynlc201603).

\section{AUTHOR CONTRIBUTIONS}

$Y Y$ and $H Y H$ designed the research. $X H Z$ and $Y Z$ performed the research. ANH finished the ISH and ICH assays. BZQ contributed analytic tools. YGW collected the samples and performed the pathology experiments. YZ followed-up the cases. ZS polished the English translation. JJZ analyzed the data and wrote the paper.

\section{ADDITIONAL INFORMATION}

Competing interests: The authors declare no competing interests.

\section{REFERENCES}

1. Chen W, Zheng R, Baade PD, Zhang S, Zeng H, Bray F, et al. Cancer statistics in China, 2015. CA Cancer J Clin. 2016;66:115-32.

2. Siegel RL, Miller KD, Jemal A. Cancer statistics, 2017. CA Cancer J Clin. 2017;67:7-30.

3. Rucci N, Angelucci A. Prostate cancer and bone: the elective affinities. Biomed Res Int. 2014;2014:167035.

4. Afshar M, Al-Alloosh F, Pirrie S, Rowan C, James ND, Porfiri E. Predictive factors for response to abiraterone in metastatic castration refractory prostate cancer. Anticancer Res. 2015;35:1057-63.

5. Zhu ZT, Jin B, Liu YP, Li YC, Lu XL, Tian X, et al. [Enhancement of all-trans retinoic acid-induced differentiation by bufalin in primary culture of acute promyelocytic leukemia cells]. Zhonghua Nei Ke Za Zhi. 2006;45:314-7.

6. Jiang $Y$, Zhang $Y$, Luan J, Duan H, Zhang F, Yagasaki K, et al. Effects of bufalin on the proliferation of human lung cancer cells and its molecular mechanisms of action. Cytotechnology. 2010;62:573-83. 
7. Chen A, Yu J, Zhang L, Sun Y, Zhang Y, Guo H, et al. Microarray and biochemical analysis of bufalin-induced apoptosis of HL-60 cells. Biotechnol Lett. 2009;31:487-94.

8. Qi F, Inagaki Y, Gao B, Cui X, Xu H, Kokudo N, et al. Bufalin and cinobufagin induce apoptosis of human hepatocellular carcinoma cells via Fas- and mitochondriamediated pathways. Cancer Sci. 2011;102:951-8.

9. Zhai XF, Fang FF, Liu Q, Meng YB, Guo YY, Chen Z. MiR-181a contributes to bufalin-induced apoptosis in PC-3 prostate cancer cells. BMC Complement Alter Med. 2013;13:325.

10. Tian HY, Yuan XF, Jin L, Li J, Luo C, Ye WC, et al. A bufadienolide derived androgen receptor antagonist with inhibitory activities against prostate cancer cells. Chem Biol Interact. 2014;207:16-22.

11. Zhang $Y$, Dong $Y$, Melkus MW, Yin $S$, Tang $S N$, Jiang $P$, et al. Role of P53-senescence induction in suppression of LNCaP prostate cancer growth by cardiotonic compound bufalin. Mol Cancer Ther. 2018;17:2341-52.

12. Yeh JY, Huang WJ, Kan SF, Wang PS. Effects of bufalin and cinobufagin on the proliferation of androgen dependent and independent prostate cancer cells. Prostate. 2003;54:112-24.

13. Yu CH, Kan SF, Pu HF, Jea Chien E, Wang PS. Apoptotic signaling in bufalin- and cinobufagin-treated androgen-dependent and -independent human prostate cancer cells. Cancer Sci. 2008;99:2467-76.

14. Wang J, Xia Y, Zuo Q, Chen T. Molecular mechanisms underlying the antimetastatic activity of bufalin. Mol Clin Oncol. 2018;8:631-6.

15. Liu T, Wu C, Weng G, Zhao Z, He X, Fu C, et al. Bufalin inhibits cellular proliferation and cancer stem cell-like phenotypes via upregulation of MiR-203 in glioma. Cell Physiol Biochem. 2017;44:671-81.

16. Zhang J, Sha J, Zhou Y, Han K, Wang Y, Su Y, et al. Bufalin inhibits proliferation and induces apoptosis in osteosarcoma cells by downregulating microRNA-221. Evid Based Complement Altern Med. 2016;2016:7319464.

17. Ding J, Yeh CR, Sun Y, Lin C, Chou J, Ou Z, et al. Estrogen receptor beta promotes renal cell carcinoma progression via regulating LncRNA HOTAIR-miR-138/200c/ 204/217 associated CeRNA network. Oncogene. 2018;37:5037-53.

18. Kartha RV, Subramanian S. Competing endogenous RNAs (ceRNAs): new entrants to the intricacies of gene regulation. Front Genet. 2014;5:8.

19. Zheng M, Liu XG, Zhou Q, Liu GL. HOTAIRM1 competed endogenously with miR148a to regulate DLGAP1 in head and neck tumor cells. Cancer Med. 2018;7:3143-56.

20. Zhao H, Han KL, Wang ZY, Chen Y, Li HT, Zeng JL, et al. Value of C-telopeptidecross-linked type I collagen, osteocalcin, bone-specific alkaline phosphatase and procollagen type I N-terminal propeptide in the diagnosis and prognosis of bone metastasis in patients with malignant tumors. Med Sci Monit. 2011;17:CR626-33.

21. Zhou Y, Wang X, Zhang J, He A, Wang YL, Han K, et al. Artesunate suppresses the viability and mobility of prostate cancer cells through UCA1, the sponge of miR184. Oncotarget. 2017;8:18260-70.

22. Chisholm KM, Wan Y, Li R, Montgomery KD, Chang HY, West RB. Detection of long non-coding RNA in archival tissue: correlation with polycomb protein expression in primary and metastatic breast carcinoma. PLoS One. 2012;7:e47998.

23. Wellberg EA, Kabos $P$, Gillen AE, Jacobsen BM, Brechbuhl HM, Johnson SJ, et al. FGFR1 underlies obesity-associated progression of estrogen receptor-positive breast cancer after estrogen deprivation. JCI Insight. 2018;3:120594.

24. Wang F, Ying HQ, He BS, Pan YQ, Deng QW, Sun HL, et al. Upregulated IncRNAUCA1 contributes to progression of hepatocellular carcinoma through inhibition of miR-216b and activation of FGFR1/ERK signaling pathway. Oncotarget. 2015;6:7899-917.

25. Kwabi-Addo B, Ozen M, Ittmann M. The role of fibroblast growth factors and their receptors in prostate cancer. Endocr Relat Cancer. 2004;11:709-24.
26. Leeming DJ, Hegele A, Byrjalsen I, Hofmann R, Qvist P, Karsdal MA, et al. Biochemical markers for monitoring response to therapy: evidence for higher bone specificity by a novel marker compared with routine markers. Cancer Epidemiol Biomarkers Prev. 2008;17:1269-76.

27. Seibel MJ. The use of molecular markers of bone turnover in the management of patients with metastatic bone disease. Clin Endocrinol. 2008;68:839-49.

28. Saad F, Lipton A. Bone-marker levels in patients with prostate cancer: potential correlations with outcomes. Curr Opin Support Palliat Care. 2010;4:127-34.

29. Alahari SV, Eastlack SC, Alahari SK. Role of long noncoding RNAs in neoplasia: special emphasis on prostate cancer. Int Rev Cell Mol Biol. 2016;324:229-54.

30. Nie Y, Liu X, Qu S, Song E, Zou H, Gong C. Long non-coding RNA HOTAIR is an independent prognostic marker for nasopharyngeal carcinoma progression and survival. Cancer Sci. 2013;104:458-64.

31. Kogo R, Shimamura T, Mimori K, Kawahara K, Imoto S, Sudo T, et al. Long noncoding RNA HOTAIR regulates polycomb-dependent chromatin modification and is associated with poor prognosis in colorectal cancers. Cancer Res. 2011;71:6320-6.

32. Bhan A, Hussain I, Ansari KI, Kasiri S, Bashyal A, Mandal SS. Antisense transcript long noncoding RNA (IncRNA) HOTAIR is transcriptionally induced by estradiol. J Mol Biol. 2013;425:3707-22.

33. Oliveira Al, Xavier-Magalhaes A, Moreira-Barbosa C, Magalhaes $H$, Henrique $\mathrm{R}_{\text {, }}$ Jeronimo $C$, et al. Influence of HOTAIR rs920778 and rs12826786 genetic variants on prostate cancer risk and progression-free survival. Biomark Med. 2018;12:257-64.

34. Chang YT, Lin TP, Tang JT, Campbell M, Luo YL, Lu SY, et al. HOTAIR is a RESTregulated IncRNA that promotes neuroendocrine differentiation in castration resistant prostate cancer. Cancer Lett. 2018;433:43-52.

35. Zhang A, Zhao JC, Kim J, Fong KW, Yang YA, Chakravarti D, et al. LncRNA HOTAIR enhances the androgen-receptor-mediated transcriptional program and drives castration-resistant prostate cancer. Cell Rep. 2015;13:209-21.

36. Gupta RA, Shah N, Wang KC, Kim J, Horlings HM, Wong DJ, et al. Long non-coding RNA HOTAIR reprograms chromatin state to promote cancer metastasis. Nature. 2010;464:1071-6.

37. Liu XH, Liu ZL, Sun M, Liu J, Wang ZX, De W. The long non-coding RNA HOTAIR indicates a poor prognosis and promotes metastasis in non-small cell lung cancer. BMC Cancer. 2013;13:464.

38. Li G, Zhang H, Wan X, Yang X, Zhu C, Wang A, et al. Long noncoding RNA plays a key role in metastasis and prognosis of hepatocellular carcinoma. Biomed Res Int. 2014;2014:780521.

39. Sethi S, Macoska J, Chen W, Sarkar FH. Molecular signature of epithelialmesenchymal transition (EMT) in human prostate cancer bone metastasis. Am J Transl Res. 2010;3:90-9.

40. Sottnik JL, Daignault-Newton S, Zhang X, Morrissey C, Hussain MH, Keller ET, et al. Integrin alpha2beta 1 (alpha2beta1) promotes prostate cancer skeletal metastasis. Clin Exp Metastasis. 2013;30:569-78.

41. Chang L, Guo R, Yuan Z, Shi H, Zhang D. LncRNA HOTAIR regulates CCND1 and CCND2 expression by sponging miR-206 in ovarian cancer. Cell Physiol Biochem. 2018:49:1289-303.

42. Hong Q, Li O, Zheng W, Xiao WZ, Zhang L, Wu D, et al. LncRNA HOTAIR regulates HIF-1alpha/AXL signaling through inhibition of miR-217 in renal cell carcinoma. Cell Death Dis. 2017;8:e2772.

43. Giri $D$, Ropiquet $F$, Ittmann M. Alterations in expression of basic fibroblast growth factor (FGF) 2 and its receptor FGFR-1 in human prostate cancer. Clin Cancer Res. 1999;5:1063-71. 